\title{
Die Wiederentdeckung der Erziehung und ihre Infragestellung durch die Neurobiologie
}

Das Suchen nach den Wurzeln von Sozialisation und Erziehung erlebt eine Renaissance. Wird den Beiträgen der halbaufgeklärten Boulevardpresse vertraut, dann melden sich die Eltern in ihrer Funktion als Erziehungsagentur zurück. Quasi im Rücken dieser neuen öffentlichen Präsenz der »Erziehung» ist ein Diskurs platziert, der die Möglichkeiten von Erziehung grundsätzlich anfragt beziehungsweise mit stark biologistisch gefärbten Tönen das humanistische Bildungsprojekt der Moderne neu zu modellieren wünscht.

Die Überlegungen in diesem Beitrag sind angeregt durch die öffentlichen Debatten und fragen schlicht nach den argumentativen Beschreibungsformeln, mit denen erstens gegenwärtig das Nachdenken über Erziehung neu angeregt wird, zweitens die Gehirnforschung, die Neurophysiologie und -biologie ihr Interesse an Phänomen des Lernen und der Bildung kommunizieren und mit denen sich drittens eine Wortmeldung prominent hervorwagt, die für eine Neubewertung des Verhältnisses von Erziehung und Genetik plädiert. Der Beitrag konfrontiert mit einem Patchwork von Impressionen, die abschließend in einem kurzen Resümee gebündelt diskutiert werden.

\section{Zur gesellschaftlichen Rückeroberung der Erziehung}

»Wir müssen unsere Kinder wieder mehr erziehen und innen Werte vermitteln. Pflichtbewusstsein, Fleiß, Aufrichtigkeit, Hilfsbereitschaft, Verlässlichkeit, Anstand, richtiges Benehmen. « Diese ermahnenden Worte stammen von keiner anderen als von der Ehefrau unseres ehemaligen Bundeskanzlers Gerhard Schröder, Doris Schröder-Köpf (2001). Um den »Erziehungsnotstand«, ausgerufen von der Fernsehmoderatorin Petra Gerster (vgl. Gerster/Nürnberger 2001), zu beenden, erfreuen sich Erziehungskurse an Volkshochschulen ebenso wie der Markt für Erziehungsratgeber und die Beratungsrubriken in den bekannten Wochenjournalen einer wachsenden Beliebtheit. 
Der gegenwärtig zu beobachtende Erziehungsboom, so ist zu vermuten, spiegelt die Erfahrungen der heutigen Elterngeneration mit ihren Kindern. Zunehmend mehr Eltern und PädagogInnen erleben die alltäglichen Aushandlungskämpfe um Grenzen, Freiräume und wiederkehrende Regel- und Absprachebrüche durch die Heranwachsenden sowie die sich darüber als notwendig herausstellenden Aushandlungsprozesse als ermüdend. Entlastend wirken da Ratschläge und Erinnerungen wie die von Doris Schröder-Köpf, die sich mit Blick auf ihre eigene Kindheit - »bis zum zehnten Lebensjahr musste ich um sieben Uhr ins Bett« - zu Strenge und Entschlossenheit bekennt und diese auch kommuniziert.

Inzwischen ist die neue Erziehungsdiskussion der Boulevardmedien auf den Seiten der sich seriös präsentierenden Feuilletons angekommen. Der Verfassungsrichter Udo di Fabio (2005) preist die neue Trias von Familie, Kirche und Selbstverantwortung - mit altpreußischen Tugenden gewürzt, wie die Schriftstellerin Tanja Dückers anmerkt - als paradigmatische Grundvokabeln einer bürgerlich verfassten Gesellschaft. Wahrnehmbaren Widerspruch erfährt er kaum. Gegen die Realität der gesellschaftlichen Prozesse von Individualisierung, Mobilitätszwang, Flexibilisierung und der Suche nach Sicherheiten in Unsicherheit unter den Bedingungen der Zuspitzung sozialer Ungleichheiten preist er die fast schon verstaubten Biedermeier-Tugenden der so genannten Wirtschaftwunderphase des letzten Jahrhunderts ebenso an, wie, der von den zeitgeistpräsenten Medien gefeierte Schüler des Reformpädagogen Hartmut von Hentig, Bernhard Bueb, seines Zeichens ehemalige Leiter des Internats »Schloss Salem«.

Bernhard Bueb kann mit Berechtigung als der Star unter den neuen ErziehungspredigerInnen angesehen werden. Neben Vorträgen in Volkshochschulen und gymnasialen Aulen, vor LehrerInnen- und Elternkongressen, vor der Kreishandwerkerschaft Heinsbergs und Auftritten in den bekannten Talk-Shows verkündet er seine erfahrungsgesättigten Thesen beispielsweise auch als Festredner in der bierdunstigen Atmosphäre der Burschenschaft »Arminia Leipzig«. Erziehung ist für Bernhard Bueb die »vorbehaltlose Anerkennung von Autorität und Disziplin«. Der »Weg zur Freiheit führt notwendigerweise durch die Unterordnung« und bedeutet »Erziehung durch Führung«. »Wer Selbstbestimmung lernen will«, so können wir vernehmen und von ihm lernen, »muss Unterordnung gelernt haben«. Eltern und LehrerInnen sollen sich wieder zu ihrem Erziehungsanspruch bekennen, Führungsqualitäten dokumentieren, sich der Infragestellung des Führungsanspruches schon durch kleine Kinder und ihrer Tyrannei widersetzen und akzeptieren, dass nur »wer führt, (...) Gefolgschaft« erwarten kann. Ordnung, Fleiß, Sauberkeit, Manieren, Disziplin und Unterordnung sind die von Bernhard Bueb verkündeten,

1 Vgl. Bueb, B. (2006a): Lob der Disziplin. Berlin. Alle nachfolgen, nicht weiter ausgewiesenen Zitate sind entweder diesem oder aber dem Text »Die Macht der Eltern schützt die Kinder« von Bernhard Bueb (2006b) entnommen. 
besser hervorgekramten Tugenden. Sie gilt es als Erziehungsprinzipien neu zu reaktivieren und mit unserer Hilfe zur »zweiten Natur« der Kinder werden zu lassen. Neuere Beobachtungen der Sozialisations- und Kindheitsforschung, ja gar der Entwicklungspsychologie sind inm fremd. Gegen den empirischen Befund der Veränderung der Generationsbeziehungen und der Aufweichung der generationalen Ordnung setzt er die schlichte Floskel »in der Pädagogik gibt es keine neuen Erkenntnisse«, lediglich Verlust, wie den von Gehorsam, der »in den letzten vierzig Jahren jedes Ansehen in der Pädagogik, aber nicht in der Armee« verlor.

Sicherlich lässt sich über die Relevanz der wieder aufgetischten Tugenden vortrefflich streiten. Vielleicht liegt in der Erinnerung an sie auch ein die Diskurse zu Fragen der Erziehung und Bildung auffrischender Esprit. Vorgetragen werden sie von den »neuen ErziehungsprophetInnen « jedoch nicht als Diskussionsanreize, sondern als zeitweise leider vergessene, »natur-gegebene« erzieherische Doktrinen. Garniert wird der neue Erziehungsboom über mehr oder weniger seriös abgesicherte Forschungsergebnisse. Schenken wir populär aufgemachten Studien unser Vertrauen, dann sind fast zwanzig Prozent aller Kinder heute als »schwierig« einzuschätzen, zeigen emotionale Störungen oder sind verhaltensauffällig. Mehr als zehn Prozent eines jeden Jahrganges werden von LehrerInnen und Eltern als »hoch gefährdet« beschrieben, weil sie beispielsweise hochgradig aggressive Verhaltensweisen zeigen oder aber als überaktiv und unruhig eingeschätzt werden (vgl. Brandau/Pretis/Kaschnitz 2003). Andere Quellen sprechen von 350.000 »behandlungsnotwendigen« Kindern (vgl. Reimann 2003). Gewalt an Schulen, in Familien und Gleichaltrigengruppen scheint explosionsartig anzusteigen - obwohl in den polizeilichen Kriminalitätsstatistiken ein solcher Trend nur schwer zu erkennen ist. Inzwischen werden selbst LehrerInnen - vermehrt selbstverständlich - Opfer von physischen und psychischen SchülerInnenattacken. An die von Heinrich Mann beschriebenen Qualen des »Professor Unrat«, der nicht nur von seiner Klasse, sondern gleich von einem ganzen Dorf malträtiert wurde, erinnern sich ebenso wenige wie daran, dass Auseinandersetzungen dieser Art seit Beginn der institutionalisierten Form von Bildung und Erziehung zum pädagogischen Alltag gehören.

Verantwortlich für die gegenwärtige »Erziehungskatastrophe« ist, wird den marktgängigsten Diagnosen gefolgt, die »Erziehungsuntauglichkeit« der heutigen LehrerInnenund Elterngeneration, weil sie, folgen wir nochmals Bernhard Bueb, zu viele »Torheiten anthropologischer und praktischer Natur « propagieren und auf die »Kräfte der Selbstbestimmung bei Jugendlichen setzen« und damit einer »Neigung zur Romantik, die unter Pädagogen durchaus verbreitet ist«, entsprechen. »Unsere Jugend« hingegen »sehnt sich nach mehr Disziplin«, obwohl sie nach der Maxime »lch. Alles. Sofort« leben, konstatiert Bernhard Bueb. Einerseits wollen Eltern und PädagogInnen perfekt erziehen, andererseits zweifeln sie jedoch an ihren eigenen Tugenden und Werten. Als ehemalige AkteurInnen des massiven Umbruchs traditioneller Wertevorstellungen in den 1970er 
Jahren und heutige GestalterInnen der Lebensqualität »Freiheit« wird innen die Kompetenz abgesprochen, den gesellschaftlichen Erfordernissen entsprechend mit den traditionellen Werten um zu gehen.

Schon vor gut zehn Jahren fand im nordrhein-westfälischen Landtag eine Debatte statt, in der insbesondere den »reformfreudigen GesamtschullehrerInnen« und »neuen« Eltern - als Apologeten der 1968er Ideologie - eine Unfähigkeit im Umgang mit den klassischen Tugenden vorgehalten wurde. Kurze Zeit später wurden die in dieser Debatte kolportierten Vorhaltungen im ansonsten nur wenig konservativen »Kursbuch« von dem Schriftsteller Peter Schneider aufgegriffen und zugespitzt: »Die Halbwüchsigen verachten die elementarsten Regeln der Fairness nicht etwa, sie kennen sie gar nicht und haben sie nie, nach den Gesetzen von Lohn und Strafe, erlernt. Sie sind nicht entmenscht und zu Bestien geworden, sie wurden erst gar nicht zu Menschen gemacht. « (Schneider 1993, S. 141) Statt erzieherisch zu handeln, so die im gleichen Beitrag vorgetragene Erkenntnis, wird nur noch erklärt und zu verstehen gesucht. Betroffenheit über desolate Lebenslagen und familiale Zusammenhänge, gleichermaßen vorgetragen von SozialhelferInnen und LehrerInnen, so Peter Schneider, entlastet diese, dem Aggressionspotential der heranwachsenden Männer erzieherisch zu begegnen - was liegt also näher, als vor dem Hintergrund dieser Diagnose die Wiederbesinnung auf die straditionellen Werte« zu empfehlen?

Der Verdacht ist nahe liegend, dass hier alle erziehungsphilosophischen und praktischen Alltagshandreichungen in einen großen Topf geworfen werden, um sie zu demaskieren. Eine solche »Gegendemaskierung« wird jedoch kaum Belege finden, denn trotz der sich hier sicherlich nicht durch Stringenz auszeichnende Präsentation von Leseerfahrungen, sind die argumentativen Strickmuster in den zitierten Überlegungen identisch. Auch Bernhard Bueb verweist auf die werte- und tugendzerstörerische Kraft der Liberalisierung und »verwirrende Psychologisierung « der Erziehungsideen in den letzten Jahrzehnten des 20. Jahrhunderts: »Die Generation nach 1968, insbesondere ihre ideologischen Vertreter interpretierten das Machtgefälle zwischen Erwachsenen und Kindern als Erfahrungsvorsprung. (...) Alle Begriffe, die an ein Machtgefälle erinnern konnten, wurden geächtet. Autorität, Gehorsam, Unterordnung und Disziplin verfielen diesem Verdikt.«

\section{Neurobiologie und Pädagogik}

Die vormoderne Erleuchtung, wonach die genetische Subjektstruktur die Wirkung pädagogischer Einflussnahmen begrenzt, wird vor diesem Hintergrund gerade zur richtigen Zeit reaktiviert (vgl. Der Spiegel 1998; Hafeneger 1998): Diejenigen, die »härteren« und traditionellen Erziehungspraxen zusprechen, werden inzwischen jedoch durch diejeni- 
gen korrigiert, die schon immer wussten, dass der Anlage und der biologischen Grundausstattung des Menschen eine größere Bedeutung zukommt als allen erzieherischen Bemühungen. Molekularbiologisch bewiesen, entwertet die Erkenntnis des »Primats der Gene« alle aufklärerisch-emanzipatorischen Bildungsprojekte. »Die Hirnforschung«, so lesen wir bei Christian Geyer (2004) im Vorwort zu dem von inm edierten Sammelband »Hirnforschung und Willensfreiheit. Zur Deutung der neuesten Experimente«, »hat sich schon immer als die hohe Schule der Illusionslosigkeit empfohlen. Der Wissenschaftshistoriker, der die aktuelle Diskussion betrachtet, dürfte von einem déjà-vu-Erlebnis ins andere fallen. Er stellt fest, dass die Loipe schon im neunzehnten Jahrhundert gespurt wurde. (...) Im Rückblick lässt sich sagen, dass Physiologie und Neurologie im Verbund mit innovativen anatomischen Konzepten die beständigsten, bis heute gültigen Beiträge zur Lokalisierungswissenschaft geleistet haben«. Ohne die vielleicht notwendige analytische Präzision möchte ich drei zentrale Befunde der jüngeren neurophysiologischen Forschung vorstellen und diskutieren.

(1) Die Neurowissenschaften liefern den Befund, dass »menschliche und tierische Gehirne sich fast in nichts unterscheiden, dass ihre Entwicklung, ihr Aufbau und ihre Funktionen den gleichen Prinzipien gehorchen«. Das experimentell validierte Wissen erlaubt sogar noch eine weitere Feststellung: „Da wir, was tierische Gehirne betrifft, keinen Anlass haben zu bezweifeln, dass alles Verhalten auf Hirnfunktionen beruht und somit den deterministischen Gesetzen physiko-chemischer Prozesse unterworfen ist, muss die Behauptung der materiellen Bedingtheiten von Verhalten auch auf den Menschen zutreffen« (Singer 2004, S. 37), da »die Nervenzellen von Schnecken (...) nach den gleichen Prinzipien wie die Nervenzellen der Großhirnrinde des Menschen« (Singer 2004, S. 39) funktionieren. Der hier durchschimmernde biologistische Naturalismus zeigt sich, und dies scheint an den präsentierten Befunden wirklich neu zu sein, offen für neue Gedankenexperimente. Zur Kenntnis genommen wird so beispielsweise die Sozialisationsforschung und das hierüber mitgeteilte Wissen, dass Aufwachsen die Herstellung von Sozialität und Kultur voraussetzt. Das »neue« Wissen wird forschungstechnisch operationalisiert und mündet in dem »empirischen« Befund, dass »wahrscheinlich alle Säugetiere adäquate Sozialisationsbedingungen bedürfen, um als Erwachsene den Anforderungen ihrer Lebenswelt gerecht zu werden« (Sachser 2004, S. 478). »Leben Hausmeerschweinchen während der Trächtigkeit in einer instabilen sozialen Umwelt, in der die Sozialpartner häufig wechseln, so verhalten sich ihre Töchter im späteren leben wie Männchen« (Sachser 2004, S. 479).

Die daraus zu ziehenden Schlussfolgerungen sind eindeutig: Allen schwangeren Frauen ist nachdrücklich zu empfehlen, sich während der Gravidität keinesfalls auf wechselnde soziale Beziehungskonstellationen einzulassen, wenn sie der Gefahr 
entgehen wollen, dass ihre Töchter später maskuline Verhaltensweisen zeigen. Aus dem ehemals »biologistischen« wird so ein »sozialer Naturalismus«, begründet mit der neurowissenschaftlichen Erkenntnis, dass »vielfältige Zusammenhänge zwischen der pränatalen Umwelt und der Gehirnentwicklung sowie dem Verhalten der Nachkommen« (Sachser 2004, S. 480) aufgrund experimenteller Studien mit Tieren nachzuweisen sind.

(2) Neurobiologische Befunde älteren und jüngeren Datums entdecken in der vermehrten Synapsenbildung in den ersten drei Lebensjahren den Grundstock für schnelle Bildungsprozesse in diesem Lebensabschnitt und begründen auch hierüber die hirnorganisch prägende Bedeutung dieses Lebensabschnittes. »Gerade die frühe Phase muss dazu genutzt werden, die gehirnbiologische Basis für spätere Leistungen und sozio-emotionale Kompetenz zu bilden« (Roth 2004, S. 516). Genau hierin artikuliert sich der Mythos der »ersten drei Jahre«, wie der amerikanische Wissenschaftsjournalist John T. Bruer mit Blick auf seine sekundäranalytischen Studien feststellt. Der Irrtum der Neurowissenschaft »besteht in der Annahme, dass es einen linearen Zusammenhang zwischen der Zahl der Synapsen im Gehirn und der Leistungsfähigkeit des Hirns beziehungsweise der Intelligenz gebe. (...) Dieses falsche Verständnis trägt viel zur Beliebtheit des Mythos bei Politikern und der Bevölkerung bei. (...) So verführerisch diese Ansicht auch sein mag, das neurowissenschaftliche Beweismaterial, über das wir verfügen, bietet keinerlei Legitimation dafür« (Bruer 2000 S. 111). Demnach »unterstützen die vorliegenden neuro- und verhaltenswissenschaftlichen Forschungsergebnisse keine ausschließliche Ausrichtung auf die ersten drei Lebensjahre bei Vernachlässigung der anderen Altersgruppen « und folglich »sollten wir uns vor Behauptungen hüten, dass (...) die wichtigste Zeit, für ein reichhaltiges und anregendes Umfeld zu sorgen, die ersten Lebensjahre sind. (...) Das, was die Neurowissenschaftler sagen, sollte allerdings unsere Skepsis gegenüber dem erhöhen, was wir in Zeitungen und im Internet lesen (...) und feststellen lassen, dass sie (...) keineswegs ein revolutionäres, auf Hirnforschung basierendes Handlungsprogramm für die Kinder gewonnen haben«. (Bruer 2000, S. 36 f.)

Gleichwohl finden PädagogInnen und PolitikerInnen gerade in den vorliegenden Ergebnissen der Gehirnforschung ihre Argumente für die Implementierung außerfamilialer Bindungsangebote für die Gruppe der unter dreijährigen Kinder. Die bildungs- und sozialpolitisch sicherlich wünschenswerte politische Steuerung der Erweiterung von bildungsorientierten Betreuungsangeboten für Kinder mit diesem Argument könnte möglicherweise wieder in Frage gestellt werden, wenn die Unsicherheit der herangezogenen Argumente erkannt wird. Mit den Belegen, die heute für den Ausbau eines bildungsorientierten Netzwerkes zitiert werden, ist schon 
morgen die Renaissance der Familie und das Zurück der Frauen an Herd und Wickeltisch zu begründen.

(3) Die Pädagogik des Lehrens und Lernens ist anfällig für neuere Entwicklungen und empirisches Wissen, das eine Effektivierung gängiger pädagogischer Praxen verspricht. Ihre jüngste Kreation verdankt sie der Neurophysiologie und begründet mit Blick auf deren Erträge die Entwicklung einer »gehirngerechten Pädagogik« (Roth 2004). Zwar wird »noch einige Zeit vergehen, bis die Methoden der Gehirnforschung soweit ausgereift sind, dass wir sie im Klassenzimmer einsetzen können«, resümiert Elsbeth Stern vom Max-Plank-Institut für Bildungsforschung. Ziel sollte allerdings sein, dass »ein Blick in das Gehirn ausreicht, um zu erkennen, ob sich ein Schüler gerade in einer Lernumgebung befindet oder ob er mit einem Unterricht gequält wird« (Stern 2004, S. 537). Die Vorstellung vom »Blick ins Gehirn« in den Klassenzimmern assoziiert schauervoll sterile Bilder. Intendiert ist, die interdisziplinäre Zusammenarbeit zwischen LehrerInnen, wissenschaftlichen FachdidaktikerInnen, Neuro- und KognitionswissenschaftlerInnen auszubauen und die Kooperationen zur Entwicklung einer »Neuropädagogik« sinnvoll zu nutzen.

Das Prestige der Neurowissenschaft und der Biogenetik ist verführerisch, selbst dann, wenn vor einer schnellen, simplifizierenden Adaption neurologischen Wissens durch die Pädagogik selbst von NeurowissenschaftlerInnen gewarnt wird (vgl. Bruer 2000, S. 52). Teile der Erziehungswissenschaft und der Pädagogik setzen jedoch auf die Wiederbelebung naturalistischer Grundannahmen, die auf Versprechen der Hirnforschung basieren, die ihre Begründerlnnen selbst häufig nicht einmal als valide Befunde kommunizieren.

\section{Wider dem »gezüchteten« Menschenpark}

Die Hoffnung, die gewaltbereiten, drogenkonsumierenden, kriminellen und »arbeitsfernen« Mitglieder der Gesellschaft durch erzieherische und bildungsorientierte, Interventionen zu sozialisieren und der Gesellschaft zu einer humaneren Physiognomie zu verhelfen, scheint insgesamt zu schwinden. Die Hoffnung an die Kraft der Aufklärung verliert an Einfluss.

Vielleicht sind die Hoffnungen trotz der diversen Katastrophendiagnosen jedoch noch nicht ganz zu verabschieden. Nicht irgendeiner, sondern der Philosoph Peter Sloterdijk, bekannt durch seine gegenaufklärerische Rekonstruktion des Zynismus (Sloterdijk 1983), gibt dem Hoffen mit einer Rede, vor einigen Jahren vorgetragen auf dem bayrischen Schloss Elmau, eine Zukunft - allerdings mit dem Preis der endgültigen Aufgabe des pädagogischen Projekts der Moderne. Die den EuropäerInnen spätestens seit der Aufklärung eigenen »Erziehungs- und Staatsreflexionen«, so erzählt uns Peter Sloterdijk 
(1999a, S. 20 f.), sind an ihr Ende gekommen, tragen sie doch zur Weigerung der Menschen bei, »die Selektionsmacht auszuüben, die sie faktisch errungen haben (...). In Zukunft wird es wohl darauf ankommen, das Spiel aktiv aufzugreifen und einen Codex der Anthropotechniken zu formulieren. Ein solcher Codex würde auch die Bedeutung des klassischen Humanismus verändern«. Inwieweit und ob »die langfristige Entwicklung auch zu einer genetischen Reform der Gattungseigenschaften führen wird (...)«. Ob die Menschheit »gattungsweit eine Umstellung vom Geburtenfatalismus zur optionalen Geburt und zur pränatalen Selektion« vollziehen können, lässt Peter Sloterdijk zwar offen. Letztendlich, auch wenn zwischen den Zeilen Zurückhaltung schimmert, votiert er für eine Anthropogenetik, in der es nicht um die »zähmende Lenkung der von sich aus schon zahmen Herden«, sondern um den Übergang von der »Menschenhütung« zur »Menschenzucht« geht, also um Selektion und die »züchterische Steuerung der Reproduktion«, so dass der »Menschenpark zur optimalen Homoöstase gelangt«.

Peter Sloterdijks Überlegungen sind schon einige Jahre alt und Paraphrasierungen seiner Thesen wie die obigen typisiert er als biologistische »Gotik und soziologische Schauerromantik«. Gewiss kann ihm nicht nachgesagt werden, offen für genetische Aussonderungen zu plädieren. Keineswegs führen inm naive Plattitüden aus der Mottenkiste der Geschichte die Feder. Aber sein verteidigender Einwurf, nicht selbst gesprochen, sondern nur den »Altvordern« Plato, Nietzsche und Heidegger und ihren »früheren Äußerungen zu modernen Stichworten« in den »toten Kellern« der Archive nachgestiegen zu sein, ist nicht ohne Weiteres zu entkräften, entlastet inn jedoch nicht. Keine noch so wohltemperierte und von weitschwelgenden Fragen umgarnte Relativierung befreit inn von der Verantwortung für den verfassten Text.

Der eigentliche Zündstoff des Sloterdjikschen »Zarathustra-Projektes« offenbart sich, wenn es von seinem philosophischen Kokon befreit und auf praktische Handlungsebenen bezogen wird. Peter Sloterdijk legt uns, den PädagogInnen, nahe, das »Scheitern« aller Humanisierungsversuche der Menschheit durch Erziehung und Bildung zu akzeptieren«, das »Versagen« auch offiziell zu bekunden und das gesellschaftliche Mandat zur Disposition zu stellen, durch gezielte Erziehungsbemühungen in der Schule und durch pädagogische Interventionen außerhalb von Schule Menschen aller Generationen größere gesellschaftliche Partizipation zu ermöglichen. Das pädagogische »Zähmungsangebot« der Pädagogik steht zur Disposition und kann nur durch die Vision eines biogenetischen »Züchtungsprojekts« ersetzt werden. Den Bildungsbereiten und -fähigen, den kognitiv, sozial und kulturell Aufmerksamen, so darf angenommen werden, steht auch zukünftig der Weg in die Bildungsinstitutionen offen. Die anderen, die »erkennbar Unerziehbaren « - das »barbarische Potential der Zivilisation « - und die alltäglich an der Gesellschaft Leidenden, bleiben dem Entwurf zufolge zukünftig von Zähmungsversuchen unbehelligt. 


\section{Unsicherheiten suchen »Verbindlichkeiten« - Resümee}

Erziehung ist ein schwieriges Geschäft. Und sie wird nicht einfacher. Konnte beispielsweise - ohne Widerspruch zu provozieren - bis vor kurzem noch angenommen werden, dass Erziehung sich zumindest noch formal durch die generationale Ordnung der Generationen konstituiert, also ältere Menschen sich Heranwachsenden mit dem Ziel zuwenden, innen die moralischen und normativen Regularien und Verständigungsformen der Gesellschaft mitzuteilen, so ist mindestens seit zwei Jahrzehnten nicht einmal mehr diese schlichte Konstante für die Entfächerung von Erziehung gegeben. Inzwischen sind es gerade die jüngeren Gesellschaftsmitglieder, die bisherige »Erziehungsverhältnisse« umkehren, in dem sie beispielsweise in den pädagogischen Institutionen und familiären Kontexten Ältere mit den Errungenschaften der Moderne bekannt machen und beispielsweise in den Umgang mit technologischen Erneuerungen einführen und so zur Etablierung neuer Lernkulturen beitragen - mit anderen Worten: Der Begriff Erziehung trägt zur Bezeichnung von pädagogischen Handlungsprozessen nur dann noch zur Verständigung bei, wenn das jeweils mit inm Gemeinte situationsbezogen präzise belegt wird (vgl. Thole 2002). Erziehung ist keineswegs eine gesellschaftlich freischwebende, beliebig begründbare Aktivität, sondern vielmehr ein bedingter und bedingender Faktor der gesellschaftlich-historischen Entwicklungs-, Auseinandersetzungs- und Entscheidungsprozesse. Das Aufpolieren traditioneller Erziehungsvorstellungen reflektiert diesen Umstand nicht. Erziehung als Medium der Reaktivierung vergangener Tugenden ist nicht nur ein »bockiges« Projekt, sondern möglicherweise eines, das von vornherein, zumindest langfristig, zum Scheitern verurteilt ist.

Eine moderne Idee von Erziehung kann die klassisch-traditionelle Differenz zwischen den »zu Erziehenden» auf der einen Seite und den aktiv »Erziehenden« auf der anderen nur noch kommunikativ und reflexiv konstituieren. Kommunikativ insofern, als dass sie auf permanente, ermüdende Aushandlungsprozesse nicht verzichten und sich nur hierüber herstellen kann; reflexiv insofern, als dass sie ihr Scheitern immer mit zu transportieren und mitzudenken hat. Erziehung unter den Bedingungen kultureller Freisetzung und gesellschaftlicher Modernisierung ist, wenn sie sich nicht selbst verleugnen will, eine Erziehung des Miteinanders und der gegenseitigen Akzeptanz, also ein intra- wie intergeneratives Projekt mit einer kaum steuerbaren Dynamik. Erziehungskurse, Elternworkshops und erzieherische Leitlinien können hierbei unterstützen, wenn sie die Einsicht in die Komplexität und Unsicherheit von Erziehung heute ansprechen. Aber Unsicherheit kann nicht rezeptförmig aufgearbeitet werden, sondern nur durch Reflexion eben dieser.

Schwierig gestalten sich für Kinder und Jugendliche wie auch für Erwachsene die Modalitäten des Aufwachsens dann, wenn die hier reklamierten reflexiven, auf gegenseitige Akzeptanz und Unterstützung vertrauenden Erziehungs- und Aushandlungsprozesse aufgrund sozialer, kultureller und insbesondere materieller Probleme und Sorgen im All- 
tag nicht permanent wachgerufen werden können. Die moderne Gesellschaft ist unsicherer geworden. Marginalisierungen und Formen der Desintegration zeigen immer deutlicher auch Formen von ausgewiesenen Exklusionen, von Ausschließungen, die sich nicht mehr und alleinig über geringe materielle Ressourcen bedingen, sondern auch Ausdruck sozial-kultureller Marginalisierungen sind. Zwischen sechs und sieben Prozent der bundesrepublikanischen Bevölkerung leben unter Bedingungen, die unterhalb des Existenzminimums liegen und damit als »arm« anzusehen sind. Darüber hinaus befinden sich zwischen $21 \%$ und $25 \%$ durchgängig in einer als prekär anzusehenden, materiell, aber auch sozial-kulturell instabilen Lebenssituation (vgl. Groh-Samberg 2005). Insbesondere diese »Prekarisierung«, in deren Folge zunehmend soziale Gefährdungen und Risiken, individuelle Problemlagen, soziale und kulturelle Verunsicherungen mithilfe der vorgehaltenen Lebensbewältigungskompetenzen nicht mehr erfolgreich bearbeitet werden können, gerät in den Reden von der »Erziehungskatastrophe« in den Blick, ohne dass sie als solche benannt wird. Nicht die Liberalisierung der den Altag rahmenden Werte durch die »Alt 68er«, sondern Trübsinnigkeit und Perspektivlosigkeit in den von materieller und kultureller Armut, Prekarisierung und Unsicherheit betroffenen sozialen Milieus provozieren die neue Aufmerksamkeit für die Bedingungen des Aufwachsens. Nicht die »Unfähigkeit« zur Erziehung ist zu dramatisieren, sondern die Unfähigkeit der Gesellschaft, die gegenwärtigen tief greifenden Exklusionen zu thematisieren. Jugendliche, die für sich keine Chance sehen, ihre Zukunft durch eine existenzsichernde, berufliche Beschäftigung abzusichern, können die Erinnerung an die klassischen Werte von Pünktlichkeit, Anstand und Disziplin womöglich hören, deren Rationalität für die eigene Lebensplanung jedoch nur partiell nachempfinden. Und Eltern, die vielleicht noch an die Bedeutung des Bildungserwerbs über schulisches Lernen vertrauten, dann aber doch erleben mussten, dass ihnen trotz aller Bemühungen keine Integration auf dem Arbeitsmarkt gelingt, denen fällt es wohlmöglich schwerer, den Erwerb von Bildungszertifikaten mittels Fleiß und Disziplin gegenüber ihren Kindern nachvollziehbar zu kommunizieren als Eltern, die die Relevanz dieser Werte in ihrer eigenen Berufs- und Lebensbiographie erfahren konnten. Mithin sind es die gesellschaftlichen Exklusionsprozesse und nicht primär die individuellen Absetzungsprozesse von den normativen Ritualen der Gesellschaft, die die gegenwärtige Rede über die elterlichen Erziehungsfähigkeiten wieder beleben - haben die »Ziele von Erziehung« keine Bedeutung mehr, weil sie gesellschaftlich enteignet wurden, verkommt die Erinnerung an Erziehung zu einer Worthülse ohne Substanz.

Erziehung in modernen Gesellschaften verlangt Mut, nicht jedoch wie Bernhard Bueb (2006, S. 17) fordert, »Mut zur Disziplin«, sondern Mut zur diskursiven Aushandlung von unterschiedlichen Auffassungen. Erziehung heute ist ein dialogischer Prozess, basierend auf gegenseitiger Akzeptanz, Toleranz, Anerkennung und Achtung. Wenn diese Grundvokabeln missachtend werden oder nicht mehr »geachtet« werden können, droht 
»Erziehung« zu scheitern. Hinweise auf neurobiologische Abläufe und altersspezifische Fähigkeiten können das Wissen und die Möglichkeiten von Erziehung und Bildung absichern, keinesfalls jedoch ersetzen. Dass ein dreijähriges Kind neurophysiologisch belegt potenziell fähig ist, mathematische Aufgaben zu lösen und sich zweisprachig zu artikulieren, bedeutet noch lange nicht, dass eine Förderung und Unterstützung dieser Möglichkeiten auch sinnvoll ist. Die gegenwärtigen Begründungsmuster, die Chancen von Erziehung und Bildung über die Erkenntnisse der Biowissenschaften neu konzipieren, unterliegen einem »naturalistischem Fehlschluss« (vgl. Zirfas 2002), indem die biologisch gegebenen Möglichkeiten von Lernen als Notwendigkeiten von Lernen ausgegeben werden und über evolutionstheoretische Argumente Fürsprache erhalten (vgl. Busche 2006).

Die Herstellung von sozialer Zugehörigkeit und das Management eines individuellen Lebensplanes stellen hochkomplexe Projekte dar, die nicht mehr entlang der in den sozialen Welten vorrätig gehaltenen Muster zu konzipieren sind. Parallel steht das Integrationsprojekt der Moderne, auch diejenigen nicht auszugrenzen und »aufzugeben«, die an der Gesellschaft scheitern oder scheitern könnten, zur Debatte - und nicht nur sozialpolitisch, sondern auch in prominenten Überlegungen zur Zukunft von Erziehung und Bildung. Zwar wird weiterhin verkündet, am Projekt einer Kompensation sozialer Ungleichheiten festzuhalten, doch wehren sich prominente VertreterInnen der Neurobiologie und auch der -pädagogik keineswegs dagegen, wenn dieses - zumindest gedanklich - zugunsten einer »Eigenschaftsplanung der Elite« (Sloterdijk 1999a, S. 21) aufzugeben kundgetan wird.

Horrorszenarien sind vielleicht unangebracht. Die umweltresistente, genmanipulierte Tomate trifft sicherlich auch nicht in naher Zukunft auf in biotechnischen Labors entwickelte, sozial verträgliche KonsumentInnen. Aber die gesellschaftlichen Unsicherheiten und die vorliegenden kulturellen Freisetzungsprozesse provozieren - wie gezeigt - Beschreibungsfolien, die im »Zurück« die Zukunft sehen. Autoritäre, auf Zucht und Ordnung setzende Erziehungsvorstellungen und pädagogische Modelle, die die Revitalisierung von Disziplin und Anpassung fordern oder die auf das unabgesicherte Wissen der Gehirnforschung und der Biogenetik vertrauen, dokumentieren nicht nur eine naturalistische Tönung und ihre Affinität zu einem zu überwunden geglaubten Biologismus, sie stellen auch nicht nur die bildungspolitische Idee der Aufklärung zur Disposition, sondern sie habitualisieren und popularisieren einen autoritär gefärbten, neoliberalen gesellschaftlichen »Klimawechsel«. 


\section{Nachtrag in forschungspolitischer Absicht}

Die Folgen dieses Klimawandels könnten auch für die sozial- und erziehungswissenschaftliche Bildungsforschung Konsequenzen zeigen. Die Frage nach ihrer Effektivität könnte Teil einer neuen Forschungsagenda sein. Dann stände auch die klassische, immer noch hoch elaborierte psychologisch motivierte Bildungsforschung und nicht nur das qualitativ-rekonstruktive und soziologische Paradigma unter Legitimitätsdruck, zumal wenn öffentlich wahrgenommen wird, wie überschaubar sich der Ertrag der Forschungen zu den schulischen Lehr-Lern-Prozessen insgesamt präsentiert und von dem langjährigen Direktor des Max-Plank-Instituts für psychologische Forschung Franz E. Weinert bilanziert wird: »Es gibt fast nichts, was (...) man nicht mit dem Unterrichtserfolg einzelner Schüler oder ganzer Schulklassen in Verbindung gebracht hätte. Die Folge davon war und ist die Inflationierung der in pädagogisch-psychologischen Untersuchungen berücksichtigten potentiellen Einflussvariabeln mit dem ebenso verallgemeinerbaren wie enttäuschenden Resultat, dass sich keine substantiellen, stabilen und generell gültigen Zusammenhänge zwischen isolierten Unterrichtsmerkmalen und den verschiedenen Erfolgskriterien der Unterrichts finden lassen. Dieser Eindruck verändert sich auch nicht, wenn statt einzelner Untersuchungen mit Hilfe statistischer Metaanalysen die Befunde aus vielen einschlägigen Studien simultan berücksichtigt werden. Eine Zusammenstellung der dabei erzielten Resultate, die sich auf 7.827 Studien und auf nicht weniger als 22.155 korrelative Beziehungen stützt (...), könnte zu der zynischen Schlussfolgerung verleiten, dass jede der berücksichtigten Variabeln in gewisser Hinsicht sowohl bedeutsam als auch unwichtig ist«. (Weinert 1989, S. 210; vgl. auch Radtke 2005; Gruschka 2004)

Der selbstkritische Rückblick evoziert die Frage nach dem Profil einer die konkrete Totalität von pädagogischen Handlungen zu evaluieren beabsichtigende, empirische Bildungsforschung. Zumindest die einseitige Favorisierung eines Forschungsparadigmas, das lediglich die Effekte von Bildungsprozessen als von ihrer Dynamik abstrahierende Momentaufnahmen (vgl. Oevermann 2004, S 473) erhebt und dokumentiert, wird hierüber kritisch angefragt. Wenn die hier diskutierten Zeitzeichen sensibel verstanden werden, dann steht auch die mit großen Datenmengen operierende, statisch-komparativ angelegte, auch mit entwicklungspsychologischem Wissen ausgestattete Bildungsforschung zur Disposition. Sie wird jedoch wohl kaum durch Forschungen kompensiert, die auf rekonstruktiv-qualitative Methodologien zurückgreifen. Neuropädagoische, molekulargenetische und gehirnbiologische Forschungsparadigmen befinden sich in Lauerstellung, um die sich mehr und mehr als unzeitgemäß darstellenden sozialwissenschaftlichen und entwicklungspsychologischen Forschungsstrategien zu verdrängen. Und sie paaren sich vortrefflich mit effizienzorientierten Bildungsideen und Erziehungsdoktrinen, 
die Disziplin, Unterordnung, Anpassung und Gehorsam durch die Führung natürlicher Autoritäten zu etablieren wünschen.

\section{Literatur}

Altenburg, M. (2006): Weniger Disziplin bitte. In: Die Zeit, 28.09.2006, Nr. 40, S. 57.

Brandau, H./Pretis, M./Kaschnitz, W. (2003): ADHS bei Klein- und Vorschulkindern. München u. Basel.

Bruer, J. T. (2000): Der Mythos der ersten Jahre. Weinheim u. a.

Bueb, B. (2006a): Lob der Disziplin. Berlin.

Bueb, B. (2006b): »Die Macht der Eltern schützt die Kinder«, http://www.faz.net/s/Rub, Zugriff am 10.10.2006.

Busche, H. (2006): Ethischer Naturalismus ohne Fehlschluss? Die Evolutionäre Ethik von Robert J. Richards, http://www.klostermann.de/zeitsch/phna_362.htm, Zugriff am 15.10.2006.

Der Spiegel (1998): Eltern ohne Einfluss. Ist Erziehung sinnlos, 1998, Heft 47.

di Fabio, U. (2005): Die Kultur der Freiheit. München.

Gerster, P./ Nürnberger, Ch. (2001): Der Erziehungsnotstand. Wie wir unsere Kinder retten. Berlin.

Geyer, Ch. (2004): Vorwort. In: Geyer, Ch. (Hrsg.) (2004): Hirnforschung und Willensfreiheit. Frankfurt a. Main, S. 9-20.

Groh-Samberg, O. (2005): Die Aktualität der sozialen Frage - Trendanalysen sozialer Ausgrenzung 1984-2004. In: WSI-Mitteilungen, 58. Jg. (2005), Heft 11, S. 616-623.

Gruschka, A. (2004): Empirische Bildungsforschung - das muss keineswegs, aber es kann die Erforschung von Bildungsprozessen bedeuten. Oder: was lässt sich zukünftig von der forschenden Pädagogik erwarten? In: Pädagogische Korrespondenz, 2004, Heft 32, S. 5-35.

Hafeneger, B. (1998): Anlage oder Umwelt. In: neue praxis, 28. Jg. (1998), Heft 6, S. 537-439.

Maag, P. (2006): Unsere Jugend sehnt sich nach Disziplin, http://www.faz.net/s/Rub, Zugriff am 10.10.2006

Mann, H. (1905): Professor Unrat. München.

Nill, A. (2006): Der Wind aus der Vergangenheit. In: Taz, 27.09.2006, S. 18.

Oevermann, U. (1996): Theoretische Skizze einer revidierten Theorie professionalisierten Handelns. In: Combe, A./Helsper, W. (Hrsg.) (1996): Pädagogische Professionalität. Frankfurt a. Main, S. 70181.

Radtke, F.-O. (2005): Pädagogen Bashing. Eine neue Runde im Streit um die Erziehungswissenschaften. Manuskript. Frankfurt a. Main.

Reimann, G. (2003): Wenn Kinder immer machen was sie wollen. Freiburg i. Breisgau.

Roth, G. (2004): Warum sind Lehren und Lernen so schwierig. In: Zeitschrift für Pädagogik, 50. Jg. (2004), Heft 4, S. 496-505.

Sachser, N. (2004): Neugier, Spiel und Lernen: Verhaltensbiologische Argumente zur Kindheit. In: Zeitschrift für Pädagogik, 50. Jg. (2004), Heft 4, S. 475-486.

Schneider, P. (1993): Erziehung nach Mölln. In: Michel, K. M./Spengler, T. (Hrsg.) (1993): Kursbuch 113. Deutsche Jugend. Berlin, S. 131-142.

Schröder-Köpf, D. (2001): »Ich musste lange um einen Ganztageskindergarten kämpfen«. In: Bildwoche, 2001, Heft 20.

Singer, W. (2004): Verschaltungen legen uns fest: Wir sollten aufhören, von Freiheit zu sprechen. In Geyer, Ch. (Hrsg.) (2004): Hirnforschung und Willensfreiheit. Frankfurt a. Main, S. 30-65.

Sloterdijk, P. (1999a): Regeln für den Menschenpark. In: Die Zeit, 1999, Nr. 38, S.15-21. 
Sloterdijk, P. (1999b): Die kritische Theorie ist tot. In: Die Zeit, 1999, Nr. 37, S. 35.

Stern, E. (2004): Wie viel Hirn braucht die Schule? Chancen und Grenzen einer neuropsychologischen Lehr-Lern-Forschung. In: Zeitschrift für Pädagogik, 50. Jg. (2004), Heft 4, S. 531-537.

Thole, W. (2002): Jugend - Freizeit, Medien und Kultur. In: Krüger, H.-H./Grunert, C. (Hrsg.) (2002): Handbuch der Kindheits- und Jugendforschung. Opladen, S. 653-683.

Weinert, F. E. (1989): Psychologische Orientierungen in der Pädagogik. In: Röhrs, H./Scheuerl, H. (Hrsg.) (1989): Richtungsstreit in der Erziehungswissenschaft und pädagogische Verständigung. Frankfurt a. Main, S. 203-214.

Zirfas, J. (2004): Die Lehre der Ethik. Zur moralischen Begründung pädagogischen Denkens. Weinheim u. Basel.

\section{Zum Autor}

Thole, Werner, Prof. Dr. phil. habil., Dipl.-Pädagoge und Dipl.-Sozialpädagoge; Hochschullehrer für Jugend- und Erwachsenenbildung am Institut für Sozialpädagogik und Soziologie der Lebensalter, Fachbereich Sozialwesen der Universität Kassel; Arbeitsschwerpunkte: Theorie und Praxis der Sozialpädagogik sowie der Kinder- und Jugendhilfe, insbesondere der außerschulischen Pädagogik mit Kindern und Jugendlichen, Professionalisierungstheorie und -forschung, Kindheits- und Jugendforschung. 